\title{
INFLUENCE OF FINING AGENTS ON GLASS MELTING: A REVIEW, PART 2
}

\author{
\#MIROSLAVA HUJOVÁ, MIROSLAVA VERNEROVÁ \\ Laboratory of Inorganic Materials, Joint Workplace of the University of Chemistry and Technology Prague, \\ Technická 5, 16628 Prague 6, \\ and the Institute of Rock Structure and Mechanics of the ASCR, v.v.i., \\ V Holešovičkách 41, 18209 Prague 8, Czech Republic \\ "\#-mail: Miroslava.Hujova@vscht.cz
}

Submitted October 12, 2016; accepted January 12, 2017

\begin{abstract}
Keywords: Glass melting, Sodium sulphate, Chemical reactions, Gas evolution, Dissolution, Fining, Foaming, Bubble nucleation

The fining agents are substances with numerous effects on glass melting. The second part of our review summarizes both the benefits and disadvantages of fining agents used in the glass industry for the purpose of degassing glass melts and removing bubbles from molten glasses. Particular attention is paid to the usage of sodium sulphate and also to the chemical and physical factors connected with such phenomena as bubble nucleation and the foaming of glass melts.
\end{abstract}

\section{INTRODUCTION}

In the first part of this review [1] a summarization of the various fining agents used in the glass melting technology was provided. Particular attention was devoted to the most widely used fining agent, sodium sulphate. This part also focused on the interactions of fining agents with glass melts, particularly batch particles dissolution, chemical reactions between batch components and fining agents and finally, their influence on the final colour of the glasses.

In the glass making industry, bubbles are not wanted in final products. Therefore, a great focus is on the identification of their origin, prevention and on finding the most effective method of the removal of gases from glass melts. If fining agents are added to the batch, large amounts of fining gases are generated during the later stages of the melting process. The released gases promote fining by their diffusion into growing bubbles. However, in such a case when a melt becomes supersaturated with dissolved gases, the new bubbles are nucleated, mostly by heterogeneous nucleation. Then the whole process of fining is prolonged and has more difficulties in maintaining the glass melt without various inhomogeneities.

In connection to fining, there are numerous works mending practical results with theory resulting in mathematical models. Their main concern is the prediction of bubbles behaviour in a glass melting space. Since these models are an effective scientific method used for the analysis, theoretical explanation and prediction of glass melting processes, we also pay a special attention to the most used models of bubble behaviour and fining.
The efficiency of the fining process depends on the redox state of the glass melts. Thus the way to optimize the fining process is to control the redox (and thus fining) through the addition of oxidizing or reducing agents [2]. Another problem is melt foaming, which slows down the glass heating and worsens the glass quality. This is caused both by bubbles from fining gases and the bubbles created by reactions between fining components and the atmosphere.

The overall purpose of our review is a brief summary and gathering of the most significant works associated with fining agents and their effects on glass melting.

Fining and its mathematical models

Bubble removal from a glass melt is traditionally called fining. Bubbles are categorized by type into primary and secondary bubbles. During the initial stage of glass melting, the most important reaction is the decomposition of carbonates. This reaction provides a massive amount of $\mathrm{CO}_{2}$, which flushes atmospheric gases out from the batch, but on the other hand, some of $\mathrm{CO}_{2}$ remains trapped within the emerging glass melt. Other gases generated in this first stage of glass melting include for example:

- $\mathrm{H}_{2} \mathrm{O}$ vapour may be present in the glass melt from batch humidity, boron sources, caustic soda and hydrated alumina,

- $\mathrm{N}_{2}$ from nitrate decomposition,

- $\mathrm{O}_{2}$ from fining agents,

- $\mathrm{SO}_{2}$ from sulphates [3]. 
However, besides the batch components, there are also other sources of gases (and bubbles) in the glass melt. In a fire melted tank, a gas source is also the fuel and its combustion [4]. Therefore, an investigation was conducted to consider the growth, shrinkage and disappearance of bubbles and their dependence on the combustion gas composition.

The study [5] claims that sulphate fining is not only efficient through the stripping of gases from the melt via $\mathrm{SO}_{2}$ bubbles growth. The author also considered a significant reduction of the surface tension, which is important for particle dissolution, bubble nucleation and foam coalescence and decay. It was proposed that sulphur retention is influenced mostly by (i) the maximum temperature to which the glass melt has been exposed, (ii) the glass basicity and (iii) the amount of atmospheric water vapour.

Primary fining from the growth of already existing bubbles is just a preliminary step for a major fining. In this stage bubble seeds are generated on non-molten sand particles, which in further melting act as nucleation centres of actual fining bubbles.

Secondary fining is then the main process, and in the case where only $\mathrm{Na}_{2} \mathrm{SO}_{4}$ is used as a fining agent, its thermal decomposition is the main source of fining gases, namely $\mathrm{SO}_{2}$ and $\mathrm{O}_{2}$. As already mentioned, these gases may diffuse into already existing bubbles, they may create new bubble seeds, react with the atmosphere or may escape through the glass level [5].

To sum up, the rate of chemical fining depends on the temperature of the melt, which affects the fining ion stability, furthermore on the glass basicity, glass viscosity and surface tension, and also gains from the exponential temperature dependence of the diffusion coefficient of the fining gas.

The fundamental reaction of the sulphate fining is the thermal decomposition, given in the form:

$$
\mathrm{SO}_{4}{ }^{2-} \leftrightarrow \mathrm{SO}_{2}(g)+1 / 2 \mathrm{O}_{2}(g)+\mathrm{O}^{2-}(l)
$$

Consequently, the intensity of the bubble removal depends on the amount of gases released by the sulphate decomposition. The shift of the sulphate equilibrium reaction to the right supports the fining process of primary bubbles, but also may produce secondary bubbles, when the melt becomes supersaturated. The increasing temperature is therefore beneficial for fining, because the given reaction is endothermic. The effect of higher temperature also enhances the decomposition of otherwise thermally stabile sulphate ions [6].

Decreasing pressure supports the fining process too, because of the low pressure inside of bubbles [7].

Also, the sulphate ions are well soluble in the basic glasses (a high concentration of alkalis) and thus these glasses are high in the gas supply. In glasses with low basicity, the fining temperatures are lower, but the gas supply is lower too [8].
It is also needed to notice that the reaction (1) is reversible and a temperature decrease or pressure increase leads to bubble shrinkage, even complete dissolution if the bubbles are small enough.

As for the oxide refining, the same principle is applied for the aspect of temperature and pressure. However, the increasing basicity destabilizes the high oxidation form and supports fining.

$$
\mathrm{Sb}^{5+}(l)+\mathrm{O}^{2-}(l) \leftrightarrow \mathrm{Sb}^{3+}(l)+1 / 2 \mathrm{O}_{2}(l, g)
$$

The reaction (2) is also reversible and bubble absorption occurs at lower temperatures, on the contrary, the bubble grows when the temperature increases. The temperature dependence of both the equilibrium constant and diffusion coefficient of oxygen has an important role in determining whether the bubble will grow or dissolve at a given temperature [9].

The work [10] studied the reversibility of gas reactions in glass melts by considering bubbles, which contained only sulphur dioxide and oxygen at the starting point. Glasses where only carbonates and nitrates were used as a source of alkali oxides showed shrinkage of bubbles at fining temperatures. The oxygen from the inside of bubbles dissolved in the surrounding melt. After the addition of a fining agent to such batches, e.g. arsenic oxide or sodium sulphate, bubbles grew and could be removed at high rates. It has been concluded as well that the behaviour of bubbles in melts was determined by the reversible reaction of oxygen with polyvalent elements present in the glass and the rate of the process was controlled by oxygen diffusion. This mechanism results in the evolution or dissolution of a given gas (and the consequential growth or shrinkage of bubbles).

The influence of oxygen on the growth and shrinkage of bubbles was also studied in [11]. Oxygen in a glass melt may be present as a molecularly dissolved element, bonded chemically via polyvalent ions or it may be trapped in gas bubbles. The work discusses the role of the present polyvalent ions. Their presence in the glass melt is responsible for the production or consumption of oxygen and consequentially for the generation, growth or dissolution of bubbles. It has been also proved that oxygen travels interstitially through the silicate network, which is important for the diffusion coefficient of oxygen in silicate melts. The diffusion rate of oxygen was considered significantly higher, when polyvalent ions were present in melt.

Nevertheless, bubble growth or dissolution depends on the concentration of all the gases present in bubbles and on their partial pressures in bubbles, $p_{i}$. Gases in the melt are expressed via $p_{i \text { melt }}$ and are dissolved either physically or as a chemical complex being in equilibrium with its physically dissolved form. The examples of almost always present physically soluble gases are Ar, $\mathrm{N}_{2}$ and partially $\mathrm{CO}_{2}$. The chemically dissolved gases are in the chemical equilibrium with their complex ions $\left(\mathrm{SO}_{4}{ }^{2-}, \mathrm{SbO}_{4}{ }^{3-}\right)$. 
Generally, the bubble starts to dissolve when:

$$
\sum_{i=1}^{n} p_{i}>\sum_{i=1}^{n} p_{i \text { melt }}
$$

and grows if reverse inequality takes place: $\sum_{i=1}^{n} p_{i}<$ $<\sum_{i=1}^{n} p_{i \text { melt }}$. The evaluation of the actual case requires knowledge of the bubble composition and gases concentration in the melt. Alternatively, the bubble growth or dissolution may be determined experimentally.

The dissolution of bubbles containing the fining gas at lower $p_{\text {imelt }}$ stops after some time because the fining gas in the bubble achieves the equilibrium with its dissolved form. However, under industrial fining conditions, the inequality expressing the bubble permanent growth is valid and the mechanism of steady bubble growth and relatively fast separation by the buoyancy force occurs. The decomposition of chemical complexes occurring at such high temperatures fulfils the condition that the $p_{i \text { melt }}$ of the fining gas itself is greater than $\sum_{i=1}^{n} p_{i}$, so the bubble growth should be permanently growing as already mentioned.

One of the possible approaches assumes that any bubble in a liquid will reach (after a sufficiently long time) the composition that is in the end independent of the initial composition and remains almost constant with time. In this case, if the pressure and temperature are given, the composition of such bubble depends only on the concentration of gases dissolved in the melt. To sum up, all the bubbles attain a constant and identical composition after a certain time, which increases with bubble radius and decreases with sinking temperature. This assumption facilitates the modelling of the fining process [12].

One of the theoretical fining possibilities is the application of the centrifugal fining. The bubble radial velocity is evolved by the rotation of glass volume and bubbles move rapidly to the centre of the rotation. However, the temperature, the size of rotating vessel and the rotation velocity must be cautiously balanced, particularly the rotational velocity. If the rotational velocity is low, the centrifugal effect is low too, if on the contrary the rotational velocity is too high, bubbles start to dissolve and their centrifuging slows down. The optimum rotation intensity must be therefore found [13-15]. The presence of the fining agent is advantageous as it prevents bubble dissolution at higher rotation velocities [16].

The connection of the above-mentioned factors brings up several models used for the theoretical explanation, the prediction of the composition and behaviour of bubbles in glass melts. This is a comprehensive topic, therefore only a few major models that are close to our research are presented.

The study [17] compares two major models where one considers particle tracing and the other model is based on the flow of the bubble phase through the melting space.
- Models tracing single bubble behaviour: This model is based on following single bubbles containing a mixture of gases which are also simultaneously dissolved in the melt. In order to describe the diffusion of the gases between the bubble and melt, their diffusion coefficients, actual and equilibrium concentration, as well as the equilibrium constants of chemically soluble gases should be known. The effect of bubbles on the melt flow is not taken into account in this model. As real bubble behaviour occurs at a rather complicated time-temperature regime, also the temperature dependencies of the mentioned quantities should be known. Precise knowledge of them is problematic as it requires extended measurements.

Also the mutual interactions of bubbles in the model of single bubbles are neglected and no information about bubble concentration in the melting space emerges from the results. Its application is convenient when the fining capacity of the melting space should be assessed by revealing the bubble critical trajectories.

- Models of a bubble distribution in a melting space e.g. model of bubble representatives traces the trajectories of selected, representative bubbles. Bubbles with a similar size and starting position may differ only slightly in terms of their trajectories. After the steady state is set up, all the bubbles are located on the trajectories of representatives. A bubble concentration field is obtained from these trajectories by the summation of bubbles in a suitable volume of the melt. Nevertheless, the bubble mutual interventions and bubble nucleation are not taken into account either.

The procedure computing simultaneously bubble composition and position may be applied for both following the fining process and behaviour of defect bubbles from different sources. As the bubbles from the defect sources differ by their initial compositions, the single bubbles of the assumed compositions are modelled, starting from the region of their anticipated arising and the effect on the resulting glass quality is evaluated.

Other than the model of bubble representatives, the convective model of bubble distribution and the analytical model of bubble distribution in channel with plug flow are also proposed. The convective model suggests that bubbles are put into the melting space as a separate phase. The resulting bubble distribution is determined by the melt convection and bubble buoyancy [18].

- The semi-empirical model [19] is based on the fact that the bubble composition at a given temperature attains a stationary state after a short time and only the fining gas is responsible for the bubble growth or dissolution. The bubble growth, usual at given temperatures, is almost linear and its values are easily measurable. Consequently, the model does not need laborious measurements of diffusion coefficients and gas solubilities. It consumes only the temperature dependences of the bubble growth 
rates and the equilibrium concentrations of the fining gas in the bubble. However, a certain model is appropriate only for the given melt. Another problem occurs during the simulation of temperature decrease, where the model describes the industrial process less accurately.

\section{Bubble nucleation}

As mentioned before, gases dissolved both physically and chemically in the melt affect the melting process. Bubbles nucleated as the primary ones arise in the stage of batch reactions and these may dissolve or grow in the melt. The condition of their dissolution or growth is given by the inequality (3) or by its reverse. However, if the concentration of a single gas in the melt, $p_{i \text { melt }}$, exceeds the external pressure, the melt becomes supersaturated and the condition for nucleation of a new bubble is fulfilled.

Nucleation could be defined as the process of clusters creation of ions or molecules which primarily occur on the present interfaces and discontinuities in melt. The consideration of dissolved gases in glass melts may also logically derive the mechanism of the interaction between specific dissolved gas species. Dissolved gases such as $\mathrm{H}_{2} \mathrm{O}, \mathrm{SO}_{2}, \mathrm{CO}_{2}$ compete in the molecular structure of the glass for the same site. So, if the glass once obtained a steady state and then underwent some significant changes (e.g. a change of temperature, pressure or composition), these changes may trigger chemical and physical reactions leading to supersaturation and subsequently to the nucleation of bubbles. In order to create a new bubble, the thermodynamic and kinetic conditions must be fulfilled to ensure a sufficient intensity of bubble nucleation [20].

If an inhomogeneity is present in the melt, the bubble nucleation is easier than in a homogeneous melt as the thermodynamic barrier of their arising is reduced. Gas supersaturation in the melt always occurs by some external stimulus, which could be a change of the gas solubility in the melt, a shift of the chemical equilibrium in favour of gas production, the electric potential, or radiation. The different ways of bubble nucleation are then defined in the terms of glass technology as a "reboil" [21].

- Compositional reboil as the consequential gas solubility decreases in the melt with changing glass composition (usually when melt basicity decreases).

- Thermal reboil as the consequence of temperature change, leading to decrease of gas solubility in the melt (temperature increase).

- Chemical reboil as the consequence of equilibrium shift to the production of a gas (fining agent, reaction with atmosphere).

- Electrochemical reboil as the consequence of rising electric potential in the melt (reaction producing oxygen).
- Radiative reboil where bubbles are produced by intensive radiation changing glass structure.

- Mechanical reboil where bubbles are produced by mechanical force (agitation, vibration) or being the consequence of existing bubbles release in the volume of melt (bubbles from refractory materials).

The mechanism of bubble nucleation is crucial in amber glass production. The equilibrium of sulphur compounds present in the melt is dependent on the furnace temperature and redox state $[22,23]$. The study for amber glasses considered a different behaviour in different types of glasses (which differed in sulphur content). As amber glasses contain more sulphur, more sulphur compounds tend to escape the glass batch and so forth, it helps to remove other trapped gases. As amber retains two types of sulphur oxidation states in a final glass, reboil is triggered by chemical reactions of sulphides and sulphates. This often results in seeds or blisters [23], when the melt is not treated under exact conditions.

Apart from the bubble nucleation and growth during the glass fining, the reboil of glass occurs in a previously bubble free glass that becomes supersaturated by a particular gas. The supersaturation of the melt may cause severe defects in the final glass. Such a phenomenon occurs mostly during the temperature rise of the melt (temperature reboil), which was already saturated at lower temperatures with a gas having the negative temperature coefficient of solubility (e.g.: $\mathrm{SO}_{2}, \mathrm{O}_{2}$ ) [4]. Fast cooling of the melt results in freeze-in bubbles due to rather low diffusion coefficients below $1250^{\circ} \mathrm{C}$. The concentration of oxygen in bubbles is important, as it may help the dissolution of bubbles; when its amount is rather low, it causes bubble entrapment within the glass melt. When using sodium sulphate, such bubbles tend to contain crystalline $\mathrm{Na}_{2} \mathrm{SO}_{4}$ and sulphur at ambient temperature as the product of the reaction where gaseous $\mathrm{SO}_{2}$ reacts to $\mathrm{SO}_{3}$ and $\mathrm{S}_{2}$ and $\mathrm{SO}_{3}$ interacts with surrounding $\mathrm{Na}_{2} \mathrm{O}$ from the glass $[4,5]$.

The composition of nucleated bubbles varies on their type and origin:

a) Bubbles of fining gas as a consequence of melt reheating.

b) Oxygen bubbles as a consequence of rising electric potential on thermocouples, refractory materials or metal surfaces.

c) Bubbles coming from the boundaries between melt and refractory materials as the consequence of decreasing melt basicity $\left(\mathrm{CO}_{2}, \mathrm{H}_{2} \mathrm{O}\right.$ bubbles, fining gas bubbles).

d) Bubbles as a consequence of a reaction between the atmosphere and melt (a reducing atmosphere or atmosphere containing water vapour reacting with sulphates producing $\mathrm{SO}_{2}$ or $\mathrm{SO}_{2}+\mathrm{O}_{2}$ ).

e) Bubbles containing $\mathrm{CO}_{2}, \mathrm{CO}+\mathrm{CO}_{2}, \mathrm{~N}_{2}$ as a consequence of a reaction between impurities either in the 
melt or in the materials (carbon, nitrides, carbides) and the glass melt.

f) Bubbles as a consequence of contact between melts with different oxidation-reduction states $\left(\mathrm{S}^{2-}\right.$, $\mathrm{SO}_{4}^{2-} \rightarrow \mathrm{SO}_{2}$ ).

All the types are frequently bound with the presence of fining agents.

Secondary fining as a technological process accompanied and characterized by bubble nucleation could be also classified in terms of the chemical and physical impulses, and both may be combined in industry as well. Chemical fining uses minor chemical components to supersaturate the melt by gases under controlled conditions and the change of glass or atmosphere composition.

This process is often controlled by the redox state of batch and by the contents of major components as it has been investigated in several works $[7,8,24]$, that focused on sulphate fining. The reduction state of glass was controlled by changes of the $\mathrm{C} / \mathrm{SO}_{4}{ }^{2-}$ molar ratio. Higher molar ratios - more reduced glasses demonstrated bubble nucleation even in the medium range of temperatures, still yielding a second generation of bubbles at high temperatures. These glass melts were often characterized by severe foaming. Bubble nucleation and sometimes also foaming enhanced sand particle dissolution, whereas the thermal efficiency of melting process was worsened. For less reduced and oxidized glasses, bubbles occurred only during the intervals of high temperatures where the thermal decomposition of sulphates took place.

Secondary fining by a physical impulse includes temperature and pressure changes, as well external forces which may influence the bubble nucleation.

When considering the application of the reduced pressure, the value of $\sum_{i=1}^{n} p_{i}$ in Equation (3) is decreased (usually reduced pressures between $10-30 \mathrm{kPa}$ are sufficient) and the nucleation is supported via melt supersaturation.

The diametrically opposite approach and thus a pressure increase may support the dissolution of bubbles. However, the total dissolution of bubbles may be technologically difficult to perform, as some gases have very low mobility and therefore high pressures are needed or a long time is required for their complete fining.

A change of temperature as an impulse for the bubble nucleation is the most usual case (thermal reboil). Temperature as the impulse for bubble nucleation is beneficial during the proper melting and fining process but damaging for an already molten and refined glass [5].

In such a case, the nucleated bubbles grow, but not all of them can be removed by rising to the boundary glass - furnace atmosphere. During the subsequent cooling, the remaining bubbles shrink as a consequence of the contraction and dissolution of the gases present. The degree of bubble shrinkage depends, however, on the exposure at high temperature [24]. If this time is rather short and no slowly diffusing gases are present in the bubble, its dissolution can be complete.

As mentioned above, reboil is generally dangerous in glass melts fined by sodium sulphate. In oxidized glass melts, fining occurs above $1450^{\circ} \mathrm{C}$ and it has been observed that bubbles in such a case could be easily soluble back into the melt. The explanation of this phenomena lies in soluble $\mathrm{SO}_{2}$ and $\mathrm{O}_{2}$ gases [5], [22] exclusively present in the given bubbles. Complete dissolution takes place during the cooling down of the melt as [5] points out. As the glass melt is cooled, the solubility of sulphates in the melt rapidly grows and, consequently, the $\mathrm{SO}_{2}$ and $\mathrm{O}_{2}$ present in the bubbles are absorbed in the melt.

The nucleation of bubbles occurs also as a consequence of refractory corrosion in contact with the melt. Subsequently, bubbles containing mostly $\mathrm{CO}_{2}$ or $\mathrm{H}_{2} \mathrm{O}$ are nucleated as a result of decreasing melt basicity. Other types of bubbles come from the pores of dissolved material. This type called mechanical reboil is not genuine nucleation because the right process is just a pore opening by corrosion. It comes, for example, from the refractory components of the gob feeders. These may also develop or worsen blisters when a cold glass is applied [23]. Bubbles from refractories are small, well dissipated in the final glass. Besides the gases present in the melt, they often consist of air residuals from a pore opening due to corrosion of the refractories. Changes in their composition with time, e.g. increasing $\mathrm{CO}_{2}$ content, often indicated their long dwell time in the glass melt [25]. Oxygen bubbles may be generated by electrolytic conduction within the refractories, particularly in $\mathrm{ZrO}_{2} /$ $\mathrm{Al}_{2} \mathrm{O}_{3}$ materials and during electrolysis of the glass melt. [4].

\section{Foaming of glass melts}

Glass foaming emerges in such a case when bubbles on the surface of melted glass survive for a longer amount time than is needed for bubbles being nucleated in the melt. Bubbles escaping from the melt are conglomerating together on the surface, being separated by lamellas. Their position is controlled by Plateau laws. According to these laws, the foam has only three lamellas intersecting at one point which is called Plateau border and they do so at an angle under $120^{\circ}$. For 3D foam, exactly 4 borders meet at one point with an angle of intersection of about $109^{\circ}$. The thickness of the Plateau borders decreases in respect to drainage influenced by gravity and viscosity. When the thickness of the lamellas sinks to about $200 \mathrm{~nm}$, drainage may stop or slow down due to lower surface tension gradients and/or depletion of surfactants. Without surface tension gradients, lamellas are not stable, and the lifespan of the foam becomes shorter. It is shortened also by the growing temperatures in a furnace $[26,27]$. Bubbles 
present at the bottom of foam layer are usually spherical, while those on the surface are polyhedral [28]. Foam height may be predicted with the help of three factors; drainage of bubbles within the foam, drainage of bubbles on the surface and the survival of drained bubbles [29]. The critical time for foam collapse decreases with higher surface tension and grows with higher viscosities [30].

In the industry, foaming is a severe problem, therefore a great deal of studies have been focused on foams and their influence on glass melting. The insulating effect of foams worsens radiative heat transfer from a combustion space - since the thermal radiation is the major part of energy supply in the glass melting. Fedorov and Pilon [23] conducted a study on the radiative properties of foam layers, and noted that due to worsened thermal radiation the glass melt temperature is also lower and thus the rates of melting and fining are limited. This results either in an increase of the dwell time for the glass melt in the furnace or the glass quality and productivity worsens $[28,31-33]$. It is also important to mention that foaming is connected to higher crown temperatures, which may result in the higher $\mathrm{NO}_{\mathrm{x}}$ emissions and also the faster refractories corrosion and the furnace wear [27, 28].

Gas release and foam formation are connected through several common important parameters which may be described as the following: the redox state of the glass melt, the water content in the melt, the temperature, the concentration of finning agent, the concentration of other dissolved gases and the stability of the glass melt lamellae in a foam layer [34, 35]. However, if we focus on sulphate fining of soda-lime-silica glass melts, we can summarize:

Above $800^{\circ} \mathrm{C}$ with the appearance of primary melt, bubbles start to be trapped within and can no longer escape through opened pores in the batch. The addition of fine cullets may also influence foaming as their melting leads to the creation of a very viscous melt covering the unreacted batch [27]. Bubbles of a primary foam contain mainly $\mathrm{CO}_{2}$ ( $\mathrm{CO}$ in reduced melts), in much lesser amounts also $\mathrm{O}_{2}, \mathrm{SO}_{2}$ and $\mathrm{H}_{2} \mathrm{O}$ vapour $[28,34]$.

Furthermore, the heating rate and particles size have a great impact on foam forming. For example, during ramp heating, the difference between fine and coarse silica is almost non-existent. However, during the isothermal treatment, which may be viewed as an extreme of ramp heating, in the batch where the fine silica was used, sulphate was melted as a phase-separated layer. Furthermore, fine silica has created an acidic melt, which enhanced an early decomposition of sulphate and thus the early generation of high foam [36].

Considering gas formation for sulphate fining under reducing conditions, sulphates and sulphides react in the thermal region of $900-1300^{\circ} \mathrm{C}$ and these reactions result in mainly $\mathrm{SO}_{2}$ foams. Then the gas formation stops and foaming decreases. The remaining sulphates decompose above $1430^{\circ} \mathrm{C}$ and a layer of secondary foam appears $[26,30,37]$.
The sulphates decomposition temperature may decrease with increasing water content in the batch owing to their mutual reaction. The optimal sulphate addition depends on its solubility at the fining zone, its loss during the first stages of melting owing to reduction reactions and on the added excess necessary for fining at high temperatures. Nevertheless, the excess sulphate addition to the batch leads to a stronger foaming tendency. Also an uncontrolled sulphur input into acidic glass, where the sulphate solubility is low, leads to foaming problems [34]. The addition of alumina, accompanied by a decrease of the sulphate solubility in molten glass has the same effect, mirrored in a decrease of the foaming onset temperature and in higher foams [27, 36, 37]. However, the addition of higher soda levels decreases foaming as it acts as a surface-active compound and increases the sulphate solubility in the melt. Every change in the redox state of melt changes the sulphate content in the melt owing to sulphate reduction reactions and thus it may cause a sudden release of gases [27].

There are also several ways to reduce the foaming of glass melts. The simplest way to destroy foams is to break the liquid films mechanically, what may be done by rotators or high pressure gas jets [38]. Spraying the melt using a solution of $\mathrm{Na}_{2} \mathrm{SO}_{4}, \mathrm{NaOH}$ or $\mathrm{KOH}$ to destabilise the foam can be also helpful, as these compounds are significantly surface active [37]. The composition and pressure of the atmosphere in the furnace is also very important parameter [38], while the quantity of foam decreases with decreasing oxygen pressure [39].

\section{CONCLUSION}

The principal effect of fining agents is their ability to release gases present in glass melts. However, their influence affects glass making immediately from the beginning of batch melting. Our review in both its parts focused on a summary of all their effects with an emphasis on sulphur compounds, which are the most commonly used in the glass making industry today. Aiming to present sulphate ability in the acceleration of melt conversion or fining at different and redox conditions, we have not omitted technologically undesired phenomena such as late bubble nucleation or glass foaming.

\section{Acknowledgement}

This work has been supported by a Student's research grant project of the internal grant agency of the Institute of Chemical Technology, Prague, and financial support from Specific University Research (MSMT No. 20-SVV/2017). 


\section{REFERENCES}

1. Hujova M., Vernerova M. (2017): Influence of fining agents on glass melting: A review, Part1. Ceramics-Silikaty, 61, 119-126. doi:10.13168/cs.2017.0006

2. Wondergem-de Best A. (1994). Redox behaviour and fining of molten glass. T. U. Eindhoven.

3. Nemec L., Kloužek J. (2003): Modelling of glass refining kinetics Part 1. Single bubbles. Ceramics-Silikaty, 47, 81-87.

4. Swarts E. L. (1986): Gases in glass. $46^{\text {th }}$ Conference on glass problems, 390-403.

5. Beerkens R. G. C. (2009): Fining of Glass Melts: What We Know About Fining Processes Today. $69^{\text {th }}$ Conference on glass problems, 13-28.

6. Arkosiova M., Klouzek J., Nemec L. (2008): The Role of Sulfur in Glass Melting Process. Ceramics-Silikaty, 52, 155-159.

7. Nemec L., Tonarova V. (2008): Glass melting and its innovation potentials: Bubble removal under the effect of the centrifugal force. Ceramics-Silikaty, 52, 225-239.

8. Klouzek J., Arkosiova M., L. Nemec, Cincibusova P. (2007): The role of sulphur compounds in glass melting. Glass Technology - European Journal of Glass Science and Technology, 48, 176-182.

9. Doremus R. H. (1960): Diffusion of Oxygen from Contracting Bubbles in Molten Glass. Journal of American Ceramics Society, 43, 655-661. doi:10.1111/j.1151-2916.1960. tb13635.x

10. Greene C. H., Platts D. R. (1968): Behavior of Bubbles of Oxygen and Sulfur Dioxide in Soda-Lime Glass. Journal of American Ceramics Society, 52, 106-109. doi:10.1111/j.1151-2916.1969.tb13351.x

11. Beerkens R. G. C., de Waal H. (1990): Mechanism of Oxygen Diffusion in Glassmelts Containing Variable-Valence Ions. Journal of American Ceramics Society, 73, 1857-1861. doi:10.1111/j.1151-2916.1990.tb05235.x

12. Nemec L., Mühlbauer M. (1980): Mathematical model of bubble behaviour during refining of glass melts. Journal of Non-Crystalline Solids, 38,593-598. doi:10.1016/00223093(80)90501-3

13. Tonarova V., Nemec L. (2008): The Bubble Behaviour under Effect of Centrifugal Force. Advanced Materials Research, 39, 475-480. doi:10.4028/www.scientific.net/ AMR.39-40.475

14. Nemec L., Tonarova V. (2005): Behavior of bubbles in glass melts under effect of the gravitational and centrifugal fields. Ceramics-Silikaty, 49, 162-169.

15. Nemec L., Tonarova V. (2006): The removal of one-component bubbles from glass melts in a rotating cylinder. Ceramics-Silikaty, 50, 216-224.

16. Tonarova V., Nemec L., Jebava M. (2010): Bubble removal from glass melts in a rotating cylinder. Glass Technology: European Journal of Glass Science and Technology, 51, 165-171.

17. Klouzek J., Nemec L., Jebava M., Trochta M., Brada J. (2007): Verification of refining models and their application. Ceramics-Silikaty, 51, 225-233.

18. Cincibusova P., Nemec L. (2012): Sand dissolution and bubble removal in a model glass-melting channel with melt circulation. Glass Technology: European Journal of Glass Science and Technology Part A, 53, 150-157.

19. Nemec L., Vernerova M., Cincibusova P., Jebava M., Klouzek J. (2012): The semiempirical model of the multi- component bubble behaviour in glass melts. Ceramics-Silikaty, 56, 367-373.

20. Hlavac J. (1981). Fundamentals of Silicate Technology. SNTL.

21. Shelby J.E. (2005), Introduction to Glass Science and Technology. Royal Society of Chemistry.

22. Klouzek J., Arkosiova M., Nemec L. (2006): Redox equilibria of sulphur in glass melts. Ceramics-Silikaty, 50, 134-139.

23. Myers D., Ross P. (2006): Amber glass - 40 years of lessons learned. $66^{\text {th }}$ Conference on glass problems, 129-139.

24. Klouzek J., Nemec L., Arkosiova M., Jebava M., Tonarova V. (2008): Determination of Gas Properties in Glass. Ceramics-Silikaty, 52, 66-71.

25. Ullrich J., Muijsenberg E. (2011): Studying bubble glass deffects that are caused by refractory materials. $71^{\text {st }}$ Conference on glass problems, 67-79.

26. Kocarkova H. (2011): Stability of glass foams: experiments at the bubble scale and on vertical film. Université Paris.

27. Beerkens R. G. C., Laimböck P. (2000): Foaming of Glass Melts. $60^{\text {th }}$ Conference on glass problems, 41-58.

28. Pilon L. (2012). Foams in Glass Manufacturing, in: Paul Stevenson (Ed.): Foam Engeering: Fundamentals and Applications. Wiley. pp. 355-409. doi:10.1002/ 9781119954620.ch16

29. Hrma P. (1990): Model for a steady state foam blanket. Journal of ColloidIinterface Science, 134, 161-168. doi:10.1016/0021-9797(90)90262-M

30. Narsimhan G., Ruckenstein E. (1986): Hydrodynamics, enrichment, and collapse in foams. Langmuir, 2, 230-238. doi:10.1021/la00068a021

31. Pilon L., Zhao G., Viskanta R. (2006): Three-Dimensional Flow and Thermal Structures in Glass Melting Furnaces: Part I, Effects of the Heat Flux Distribution. Glass science and technology, 75, 55-68.

32. Fedorov A. G., Viskanta R. (2000): Radiation Characteristics of Glass Foams. Journal of American Ceramic Society, 76, 1313-1320. doi:10.1111/j.1151-2916.2003.tb03468.x

33. Hrma P. (2009): Effect of heating rate on glass foaming: Transition to bulk foam. Journal of Non-Crystalline Solids, 355, 257-263. doi:10.1016/j.jnoncrysol.2008.11.007

34. Beerkens R. G. C., Van Der Schaaf J. (2006): Gas release and foam formation during melting and fining of glass. Journal of American Ceramic Society, 89, 24-35. doi:10.1111/j.1551-2916.2005.00691.x

35. Van Der Schaaf J., Beerkens R. G. C. (2006): A model for foam formation, stability, and breakdown in glass-melting furnaces. Journal of Colloid Interface Science, 295, 218-229. doi:10.1016/j.jcis.2005.07.068

36. Kim D., Hrma P. (1992): Foaming in glass melts produced by sodium sulfate decomposition under ramp heating conditions. Journal of American Ceramic Society, 75, 2959-2963. doi:10.1111/j.1151-2916.1992.tb04371.x

37. Laimböck P. (1998). Foaming of glass melts. Technische Universitet Eindhoven.

38. Cable M., Rasul C.G, (1967): Spontaneous Bubble Formation in Silicate Melts at High Temperatures: II, Effect of Wet Atmospheres and Behavior of Mixed Alkali Glasses. Journal of American Ceramic Society, 50, 528-531. doi:10.1111/j.1151-2916.1967.tb14988.x

39. Rasul C.G., Cable M. (1966): Spontaneous bubble formation in Silicate melts at high temperatures. Journal of American Ceramic Society, 49, 568-571. doi:10.1111/j.1151-2916.1966.tb13165.x 